\title{
ÉTUDES SUR LA SUPPRESSIVITÉ DES MUTANTS A DEFICIENCE RESPIRATOIRE DE LA LEVURE
}

\section{EXISTENCE AU NIVEAU CELLULAIRE DE DIVERS "DEGRÉS DE SUPPRESSIVITÉ" *}

\author{
BORIS EPHRUSSI† et SIMONE GRANDCHAMP \\ Laboratoire de Génétique Physiologique du CNRS, Gif-sur-Yvette
}

Received I o.vi.64

ON sait que les souches de mutants cytoplasmiques à déficience respiratoire, "petites végétatives", de la levure Saccharomyces cerevisia peuvent, d'après le comportement dans les croisements avec la levure normale, être classées en deux catégories: petites neutres et petites suppressives, ces dernières imposant le caractère de petite aux zygotes avec des fréquences qui définissent leur degré de suppressivité (Ephrussi et al., 1954).

L'existence établie de souches petites neutres, d'une part, et hautement suppressives, d'autre part, a conduit à se demander si les souches à degrés de suppressivité intermédiaires sont des populations mixtes de ces deux types cellulaires en proportions variées (hypothèse I) ou si elles représentent, au contraire, des populations homogènes dont chaque cellule n'a qu'une probabilité limitée de suppression (hypothèse 2) (Ephrussi et al., I954 et 1955). L'examen de la nature de la descendance végétative immédiate des zygotes formés dans les croisements entre levure normale $(G)$ et diverses souches petites $(p)$, semblait apporter un appui à la seconde de ces hypothèses (loc. cit.). On a en effet constaté, d'une part, que ces zygotes peuvent être classés en trois catégories: (a) zygotes à descendance entièrment $\mathrm{G} ;(b)$ zygotes à descendance entièrement $p ;(c)$ zygotes à descendance mixte; et, d'autre part, que la fréquence relative de ces types de zygotes dans un croisement donné, reflète le degré de suppressivité du parent $p$. Alors que dan: les croisements $\mathrm{G} \times p$ neutre et $\mathrm{G} \times p$ hautement suppressive, presque tous les zygotes appartiennent aux types $(a)$ et $(b)$ respectivement, ils sont des trois types dans les croisements $\mathrm{G} \times p$ moyennement suppressive. Il en ressort qu'il existe au niveau cellulaire, au moins trois types de petites, mais ce genre d'expériences ne permet pas d'établir s'il y en a de plus nombreux.

L'objet du présent travail est de décrire des expériences de clonages successifs qui mettent en évidence une forte corrélation entre le degré de suppressivité d'une cellule et celle de sa descendance végétative et par conséquent permettent de conclure qu'il existe, sur le plan cellulaire, plus de trois types de petites.

* Ce travail a bénéficié d'une subvention de la Fondation Rockefeller.

$\dagger$ Adresse actuelle: Western Reserve University, Cleveland, Ohio, U.S.A. 
Une telle conclusion était suggérée déjà par des expériences effectuées, dans notre laboratoire, en 1957 par R. Milkman, (inédit). Cependant, conduites sur une échelle trop petite pour être entièrement convaincantes, et avec une souche de levure G qui contenait un pourcentage elevé de $p$, elles nous ont paru mériter une investigation plus étendue.

\section{MATERIEL ET TECHNIQUES}

Toutes les souches utilisées dans les expériences ci-dessous sont dérivées des clones haploides $\mathrm{C}_{982-19 b}$ et $\mathrm{C}$ 982-ıd dont l'origine a été décrite par Ephrussi et al. (1954). Ce sont:

(a) C 982-ı $g b-1 a$, souche à respiration normale (G), à faible fréquence de mutation en $p$. Elle a été sélectionnée à la suite d'une série de passages de la souche C $982-19 b$ sur milieu complet à faible teneur en glucose $(0,2$ pour cent.). Les populations de C $982-$ I $9 b$-I $a$ ne contiennent d'habitude que I à 2 pour cent. de $p$, alors que la souche mère en contient de Io à 20 pour cent. C'est une souche diploïde homozygote pour le signe $a$ et exigeant l'uracile et la méthionine. On ne sait pas si la faible fréquence de mutants $p$ de cette lignée est liée à sa diploïdie.

(b) C 982-19dp 6/6, clone haploide $p$, de génotype: $\alpha ; t r i_{1} ; h i_{1}$. Isolé en 1953 (Ephrussi et al., 1954), ce clone présentait à l'époque un degré de suppressivité d'environ 50 pour cent. Au moment de l'exécution de ce travail son degré de suppressivité variait entre 25 et 40 pour cent.

(c) I9dp 6/6 M I5-1B-2/16, et $(d)$ I9dp 6/6-12, isolées de la précédente en $195^{8}$ et 1959. Ces souches ont subi de nombreux repiquages depuis leur isolement et, au moment des expériences décrites ici, présentaient respectivement des degrés de suppressivité d'environ 95 et 45 pour cent.

Les cultures stock ont été conservées à la glacière sur milieu YPGA (Yeast ex tract Difco 1 pour cent.; bacto-peptone 2 pour cent.; dextrosol 2 pour cent.; sulfate d'adénine o,oo4 pour cent.) gélosé à 2 pour cent.

La technique de croisement et le milieu pour la détection des prototrophes sont ceux décrits par Jakob (1962). Le milieu était cependant tamponné à $p \mathrm{H}=6,4$ à l'aide de tampon préparé de la façon suivante: on mélange en quantités égales des solutions molaires de $\mathrm{PO}_{4} \mathrm{HNa}_{2}, I_{2} \mathrm{H}_{2} \mathrm{O}$; de phtalate acide de $\mathrm{K}$ et d'acide succinique, puis on ajuste le $p \mathrm{H}$ à 6,4 à l'aide d'une solution molaire de $\mathrm{KOH}$. Ce tampon est utilisé à une cocentration finale de o, I $M$. Tampon et solution de glucose ont toujours été additionnés après stérilisation.

Le clonage des souches $p$ a été fait par étalement sur milieu YE o,5 (Yeast extract Difco 0,5 pour cent.; dextrosol o,5 pour cent.).

Enfin la détermination du pourcentage de $p$ spontanées dans la souche $G$ est faite par étalement sur milieu YE G o, I (Yeast extract Difco o,5 pour cent.; glycérol 3 pour cent. vol.; dextrosol 0,1 pour cent.).

Toutes les expériences ont été effectuées à $30^{\circ} \mathrm{C}$.

\section{DETERMINATION DU DEGRE DE SUPPRESSIVITÉ}

Le degré de suppressivité, pour cent. $\mathrm{S}$, a été mesuré par le pourcentage de colonies blanches $(p)$ établi après étalement du mélange de copulation sur milieu minimun (où seuls les zygotes prototrophes peuvent proliférer) et coloration au chlorure de tétrazolium.* Cette coloration, appliquée aux colonies après quatre jours et demi d'incubation à $30^{\circ} \mathrm{C}$, , est effectuée selon la technique d'Ogur, St.

* Afin de tenir compte de la présence de quelques $p$ dans la souche $G$, la valeur de pour cent. $S$ est calculée par la formule: pour cent. $S=\frac{(X-Y)}{100-Y} \times 100$. Cette correction est suffisante lorsque $\mathrm{n}$ est petit. (X: pour cent. zygotes à descendance "petite" dans le croisement; Y: pour cent. $p$ spontanées dans $\mathrm{G}$.) 
John et Nagai (1957) légèrement modifiée (addition au réactif de o, I pour cent. de dextrosol). On notera qu'appliquée aux colonies dont il s'agit ici, elle révèle l'existence, à côté de colonies typiquement $\mathrm{G}$, se colorant en rouge et de $p$ blanches, de colonies partiellement colorées dont la fréquence dépend de la suppressivité du parent $p$ utilisé dans le croisement (Ephrussi et al., inédit). Cependant la fréquence des colonies enièrement blanches constitue la seule mesure objectivc du degré de suppressivité et a donc été utilisée dans ce travail.

Les erreurs affectant les déterminations de pour cent. S sont très faibles, les comptages ayant porté dans chaque cas sur un millier de colonies.

\section{RESULTATS EXPERIMENTAUX}

Une première série d'expériences a eu pour point de départ deux clonages de la souche petite $\mathrm{C}$ 982-19dp 6/6. Trente six heures après le clonage, c'est-à-dire après vingt générations cellulaires environ, une partie de chaque clone (colonie) a été subclonée et l'autre utilisée pour la détermination du degré de suppressivité moyen du clone. Les résultats de cette première détermination sont donnés au niveau $\mathrm{A}$ du graphique I. On voit que la souche de départ (quoique ultimement d'origine monocellulaire) a fourni des clones répartis en une gamme continue de degrés de suppressivité. Ceci pourrait être dû au grand nombre de repiquages qu'elle a subi depuis son isolement et serait une nouvelle illustration des variations que l'on observe au cours de la propagation d'un clone (Ephrussi et al., 1954).

Les subclones de 8 clones du niveau $\mathrm{A}$, dont les degrés de suppressivité étaient respectivement de $0,1,10,20,25,55,70$ et 95 pour cent. ont été étudiés par la même technique que ci-dessus. L'examen des résultats portés sur le graphique (niveau B) fait apparaître très nettement l'existence d'une corrélation mère-fille. Cette corrélation est très forte aux deux extrémités de l'échelle de suppressivité, c'est-à-dire pour les $p$ neutres et les $p$ très hautement suppressives. Elle est beaucoup plus faible sur le reste de l'échelle de suppressivité (en particulier dans la région 20 à 30 pour cent. $S$ ), ce qui aboutit à une dispersion considérable des valeurs de pour cent. $S$ à l'intérieur de chaque famille de subclones. (La très forte corrélation mère-fille pour les degrés de suppressivité très élevés est particulièrement bien illustrée par le clonage de la souche 2/1 6 figurée en blanc sur le graphique 1 .)

Afin d'obtenir des renseignements sur la nature de ces variations, nous avons, dans la suite de cette série d'expériences, soumis au subclonage non seulement des clones représentant le mode, mais encore caux des extrémités de la distribution "familiale". L'analyse des résultats des deuxième et troisième subclonages que l'on peut lire aux niveaux $\mathrm{C}$ et $\mathrm{D}$ du graphique $\mathrm{I}$ : $(a)$ confirme l'existence d'une corrélation inère-fille et de ses variations le long de l'échelle de suppressivité; $(b)$ montre que le subclonage d'un clone de suppressivité $x$, représentant l'extrémité d'une distribution "familiale ", fournit une 
famille de subclones dont la valeur modale est tantôt $x$ ou une valeur voisine, tantôt sensiblement identique à celle de la famille dont le clone extrême est issu.

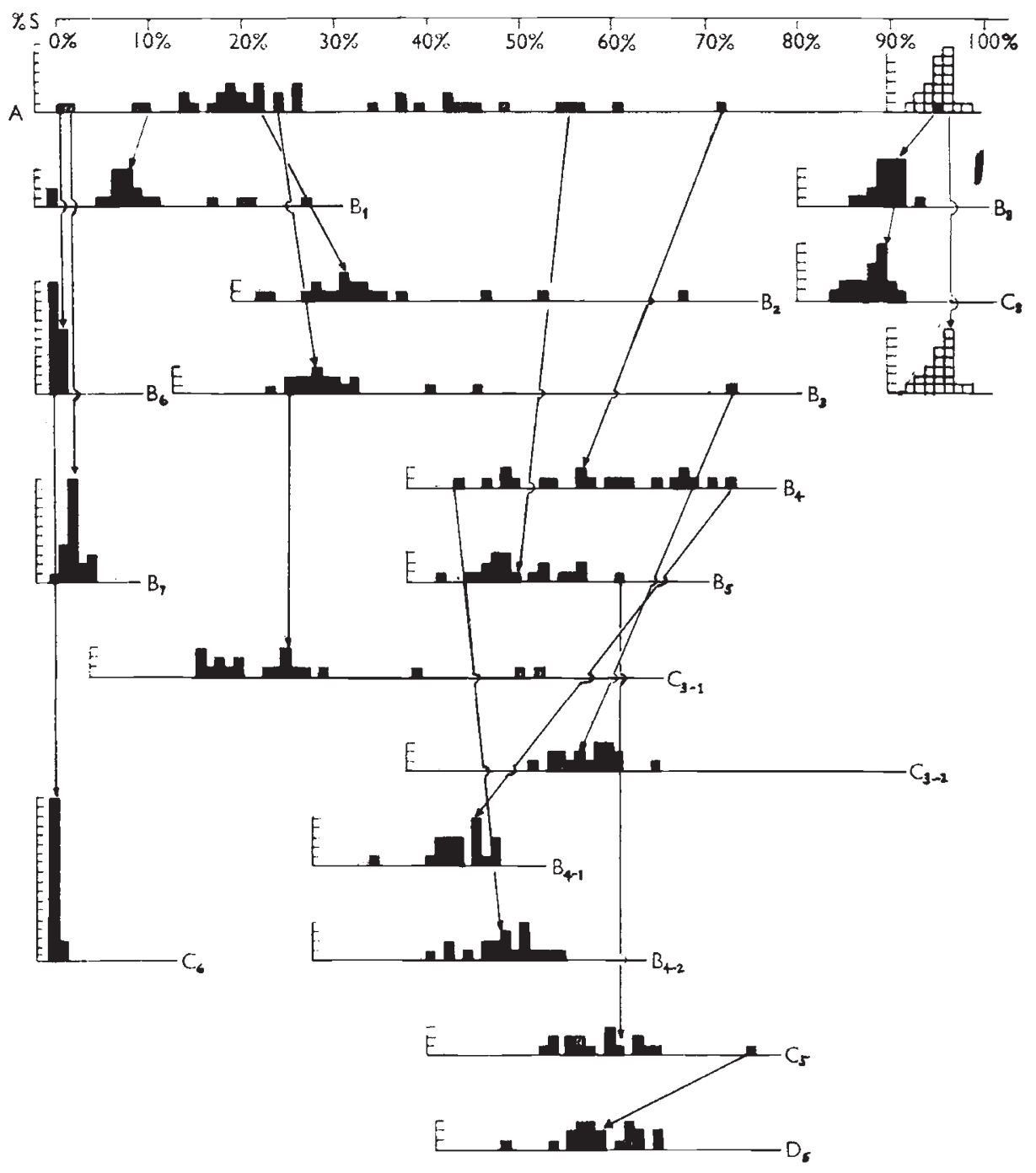

Graphique 1.-Clonage de la souche C 982 ig $d p 6 / 6$ (niveau A) et subclonages pour différentes valeurs du pour cent. $S$ (niveaux $B, C$ et $D$ ) $=1$ clone.

Clonage de la souche $\mathrm{C}_{982} 19 d p 6 / 6$ iB $2 / 16$ (A) et subclonage (B). $\square=1$ clone.

Une deuxième série de subclonages, effectués de la même manière,* a eu pour origine la souche $19 d p 6 / 6-12$. Les résultats obtenus sont donnés dans le graphique 2 . On voit qu'ils confirment essentiellement ceux de la série précédente, à ceci près que les quatre valeurs extrênes des distributions "familiales" testées ont été toutes transmises à leur descendance.

\footnotetext{
* Voir cependant note ** page suivante.
} 


\section{DISCUSSION}

Le présent travail montre tout d'abord que l'hypothèse I iselon laquelle un degré intermédiaire de suppressivité est la résultante des propriétés de deux types cellulaires d'une population mixte) n'est pas soutenable: en effet les clonages des souches à degrés de suppressivité intermédiaires n'ont pas permis de mettre en évidence la présence de la proportion de $p$ neutres requise par cette hypothèse.

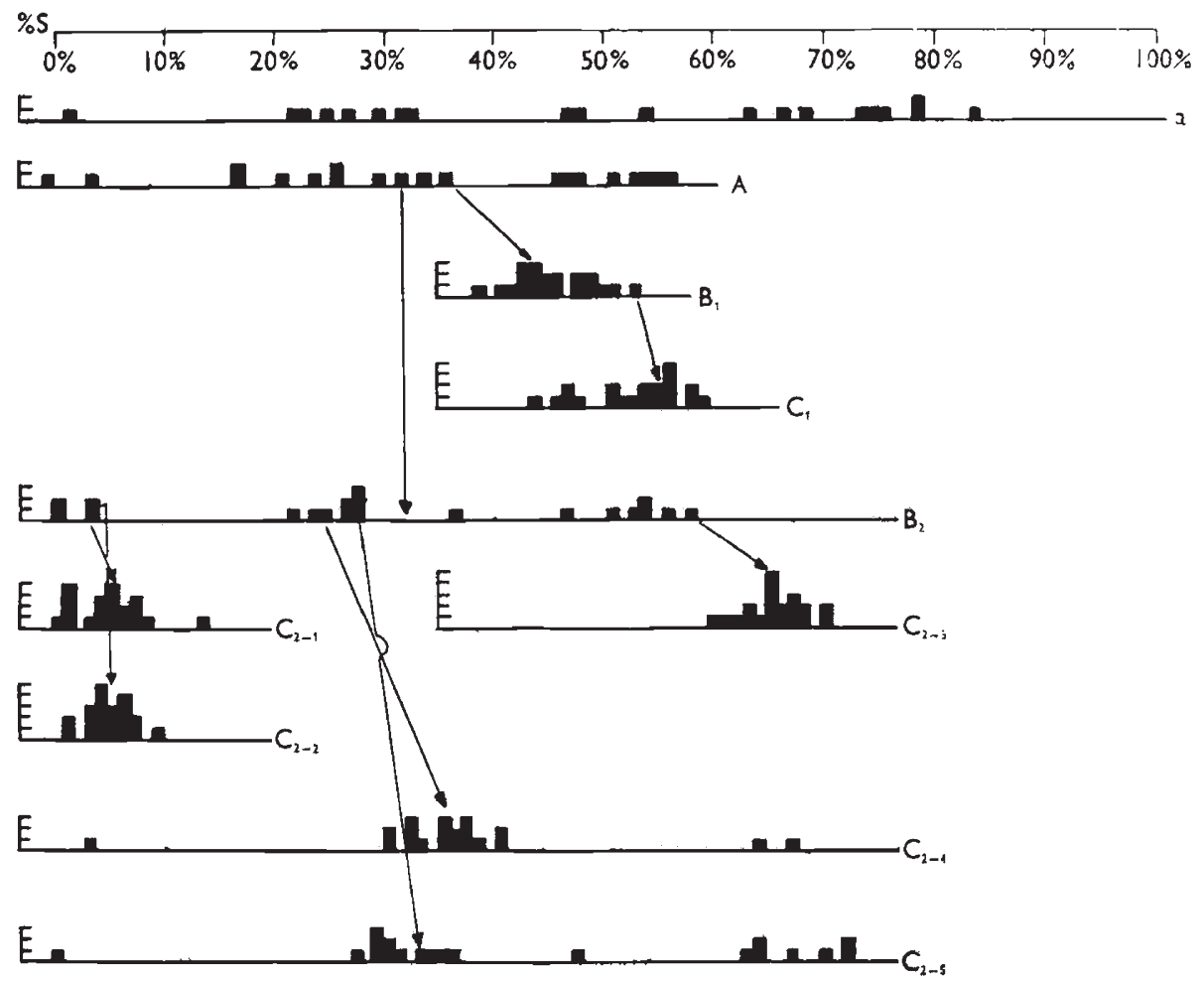

Graphique 2.-Clonage de la souche C 982 ig $d p$ 6/6-12 (a) sur milieu préparé $4^{8}$ h. à l'avance, (A) Sur le mème milieu conservé pendant des temps variables.

Subclonages pour différentes valeurs du pour cent. $\mathrm{S}$ (niveaux $\mathrm{B}$ et $\mathrm{C}$ ). $\mathbf{D}_{\text {a }}=$ I clone.

Par ailleurs, l'ensemble des résultats décrits, en révélant une corrélation nette entre le degré de suppressivité d'un clone donné et celui des clones des "générations" précédentes, quel qu'ait été le point de départ sur l'échelle de suppressivité, prouve l'existence, sur le plan cellulaire, sinon d'une gamme continue, du moins de très nombreux types de $p$ à degrés de suppressivité variés, prévus par l'hypothèse 2.*

Ces divers types de petites, quoique relativement stables au cours

- Les degrés de suppressivité représentent donc une propriété cellulaire et non une caractéristique des populations. Ce fait met en doute le mécanisme suggéré dans un travail antérieur (Ephrussi et al., 1955) de la formation de deux sortes d'asques ( $4 p: 0 \mathrm{G}$ et op:4G), selon le moment de la sporulation, à la suite des croisements $G \times p$ hautement suppressive. 
de la multiplication végétative, donnent cependant constamment naissance à des " variants " qui diffèrent par leur degré de suppressivité du clone d'origine. On a vu en effet que les valeurs extrêmes fournies par certains clones d'une famille de clones à forte dispersion ne sont dues que pour une faible part à des variations d'expression non transmissibles, c'est-à-dire dues à l'action de facteurs aléatoires.*

La majorité des variations extrêmes sont au contraire transmises d'une manière stable; on en voit plusieurs exemples sur les graphiques I et 2 .

La question du mécanisme de ces variations fait partie du problème général de la base génétique de la suppressivité. Il paraît établi que la nature neutre ou suppressive d'une petite, quelque soit son degré de suppressivité, n'est pas liée à la ploïdie de la cellule. En effet des croisements entre une même $\mathrm{G}$ (qu'elle soit haploïde ou diploïde homozygote pour le signe) et des $p$ haploïdes neutres ou de suppressivités variées donnent une ségrégation normale de 5 marqueurs (Ephrussi et al., inédit). Par ailleurs il a été montré précédemment (Ephrussi et al., I 954 et I955) que le phénomène de suppressivité repose sur un processus actif, dû à la présence dans les $p$ suppressives de ce que nous avons appelé le facteur suppressif (FS). La nature de ce dernier demeure inconnue. Si l'on sait qu'il est localisé dans le cytoplasme (l'étude de Wright et Lederberg (1957) a en effet montré son passage par le cytoplasme dans les hétérocaryotes), on ne connaît guère le lieu de sa formation (cytoplasme ou noyau) et on sait encore moins si les différents degrés de suppressivité correspondent à des variations qualitatives ou quantitatives du FS et comment est assurée leur stabilité. Toute hypothèse concernant la nature du FS devra tenir compte de la multiplicité, sur le plan cellulaire, des degrés de suppressivité démontrée dans ce travail, ainsi que du fait que les clones à pour cent. $\mathrm{S}=20$ à 40 constituent la classe dont la variabilité est la plus forte.

Une dernière conclusion s'impose ici. L'homogénéité des subclones de haute suppressivité permet d'affirmer que toutes les cellules d'un clone donné contiennent essentiellement le même FS. Si donc, à la suite du croisement d'un tel clone avec une souche $G$, on observe la formation de plus d'un type de colonie, en particulier des $\mathrm{G}$ et des $p$, cela ne peut être dû à ce que seule une fraction des zygotes a reçu le FS. Le même argument s'applique aux $p$ de suppressivité basse. A première vue une telle conclusion ne peut pas être étendue aux clones dérivés d'une $p$ de suppressivité moyenne du fait de la grande dispersion de ceux-ci et de la production fréquente de variants stables. Et cependant, le fait que, abstraction faite des quelques variants, les clonages successifs d'une $p$ de suppressivité moyenne donnent naissance à des familles de clones présentant non seulement la même suppressivité moyenne, mais encore une dispersion sensiblement identique, montre

\footnotetext{
- Nous avons trouvé récemment que la dispersion discutée, ainsi que les valeurs de pour cent. S, sont significativement réduites si les étalements sont faits sur des milieux préparés $4^{8} \mathrm{~h}$. à l'avance (comparer lignes a et A du graphique 2).
} 
bien qu'ici encore les descendants d'une $p$ contiennent tous le FS et que ce dernier, par ces propriétés, est étroitement apparenté à celui du clone d'origine.

La présence du FS dans toutes les cellules d'un clone, quel que soit son degré de suppressivité, montre, somme toute, que le degré de suppressivité exprime la probabilité de la conversion en $p$ des zygotes des croisements $\mathrm{G} \times p$ suppressive.

\section{RÉSUMÉ}

L'objet du présent travail était d'établir si les degrés de suppressivité intermédiaires, caractéristiques de certaines souches "petites" de levure, (a) sont des propriétés de populations mixtes, composées de petites neutres et de petites hautement suppressives ou, au contraire, (b) se retrouvent sur le plan cellulaire.

Des clonages successifs, en révélant une forte " corrélation mèrefille", sont en accord avec la seconde hypothèse.

\section{SUMMARY}

The purpose of the present work was to establish whether the intermediate degrees of suppressiveness characteristic of certain strains of "petites" (a) are due to the fact that these strains represent mixed populations composed of neutral and highly suppressive petites, or $(b)$ are directly due to the intermediate character of the individual cell.

Cloning experiments, by revealing in particular a strong " motherdaughter correlation ", support the second hypothesis.

Nous remercions le Dr F. Sherman de l'aide qu'il a apportée dans l'exécution de quelques-unes des expériences décrites dans ce travail.

\section{BIBLIOGRAPHIE}

EPHRUSSI, B., MARGERIE-HotTINGUER, H. DE, ET ROMAN, H. I954. Sur le comportement génétique des mutants à déficience respiratoire de la levure. Bème Congrès Int. Bot., II I-1 20.

EPHRUSSI, B., MARGERIE-HOtTINGUER, H. DE, ET ROMAN, H. 1955. Suppressiveness: a new factor in the genetic determinism of the synthesis of respiratory enzymes in yeast. Proc. Natl. Acad. Sci., US, 4I, 1065-1071.

JAKOB, H. 1962. Technique de synchronisation de la formation des zygotes chez la levure S. cerevisia. C. R. Acad. Sci., 254, 3909-39 II.

OGUR, M., ST. JOHN, R., ET NAGAI, s. I957. Tetrazolium overlay technique for population studies of respiration deficiency in yeast. Science, 125, 928 .

WRIGHT, R. E., ET LEDERBERG, J. 1957. Extranuclear transmission in yeast heterokaryons. Proc. Natl. Acad. Sci., US, 43, 919-923. 